\title{
Xpert MTB/RIF Ultra: innovación en el diagnóstico de la tuberculosis
}

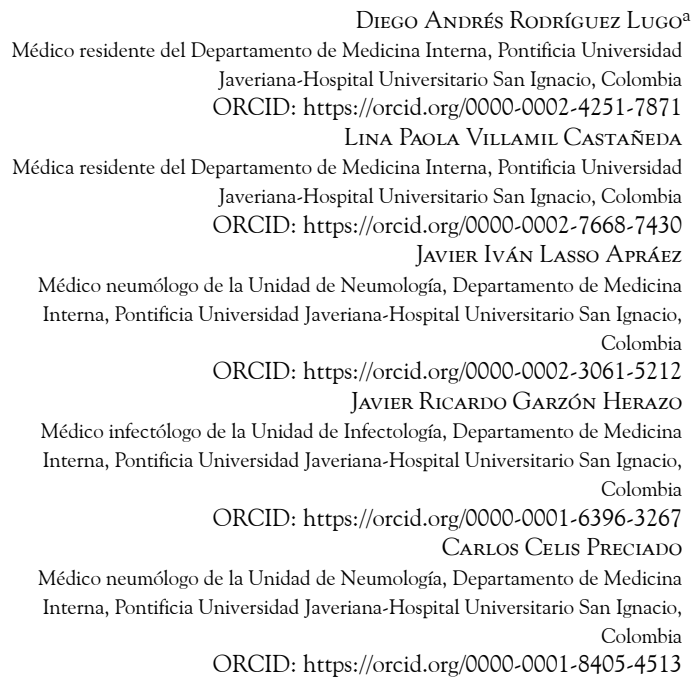

Diego Andrés Rodríguez Lugo Médico residente del Departamento de Medicina Interna, Pontificia Universidad Javeriana-Hospital Universitario San Ignacio, Colombia ORCID: https://orcid.org/0000-0002-4251-7871 Lina Paola Villamil Castañeda Médica residente del Departamento de Medicina Interna, Pontificia Universidad Javeriana-Hospital Universitario San Ignacio, Colombia ORCID: https://orcid.org/0000-0002-7668-7430 JaVier Iván Lasso Apráez Médico neumólogo de la Unidad de Neumología, Departamento de Medicina Interna, Pontificia Universidad Javeriana-Hospital Universitario San Ignacio,

Colombia

ORCID: https://orcid.org/0000-0002-3061-5212 Javier Ricardo Garzón Herazo Médico infectólogo de la Unidad de Infectología, Departamento de Medicina Interna, Pontificia Universidad Javeriana-Hospital Universitario San Ignacio, Colombia ORCID: https://orcid.org/0000-0001-6396-3267 Carlos Celis Preciado Médico neumólogo de la Unidad de Neumología, Departamento de Medicina Interna, Pontificia Universidad Javeriana-Hospital Universitario San Ignacio, Colombia

ORCID: https://orcid.org/0000-0001-8405-4513

a Autor de correspondencia: dandres_rodriguez@javeriana.edu.co

Cómo citar: Rodríguez Lugo DA, Villamil Castañeda LP, Lasso Apráez JI, Garzón Herazo JR, Celis Preciado C. Xpert MTB/RIF Ultra: innovación en el diagnóstico de la tuberculosis. Univ. Med. 2021;62(1). https://doi.org/10.11144/Javeriana.umed62-1.xper

\section{RESUMEN}

La tuberculosis (TB) es una enfermedad infecciosa con alta mortalidad en el mundo, y por ello se considera un problema de salud pública. La dificultad para su diagnóstico, sobre todo en países de bajos y medianos ingresos, limita su erradicación. El Xpert MTB/RIF, que es una prueba molecular, provee un diagnóstico rápido e informa sobre la presencia de cepas resistentes a la rifampicina, lo que permite iniciar un tratamiento oportuno y eficaz. Sin embargo, tiene problemas para el diagnóstico de casos paucibacilares y se han reportado falsos positivos en cuanto a la resistencia a rifampicina y en pacientes con antecedente de TB. Por esto, ha aparecido el Xpert MTB/RIF Ultra, que ha mostrado mejor sensibilidad para el diagnóstico de la TB, sobre todo en casos paucibacilares, y disminución de falsos positivos para resistencia a rifampicina, siendo la prueba molecular más recomendada por la Organización Mundial de la Salud. Teniendo en cuenta lo anterior, se espera que mejore el diagnóstico de la enfermedad y que dé la oportunidad de iniciar el tratamiento de forma oportuna. Con una revisión de la literatura se buscaron los aspectos por mejorar en el diagnóstico de la tuberculosis, características y rendimiento del Xpert MTB/RIF y Xpert MTB/RIF Ultra para formas pulmonares y extrapulmonares y para la detección de la resistencia a la rifampicina.

Palabras clave

tuberculosis; tuberculosis pulmonar; técnicas de diagnóstico molecular; rifampicina; Xpert MTB/RIF.

\section{ABSTRACT}


Tuberculosis (TB) is and infectious disease with a high mortality and is a great challenge for public health worldwide. Among the problems for its eradication, diagnosis represents a major challenge, particularly in lowand middle-income countries. The Xpert MTB/RIF is an automated molecular test; it yields a rapid diagnosis and screens for the presence of rifampin resistance, permitting an early and efficient treatment. However, it has a low sensitivity in patients with paucibacillary disease, and false positives for rifampin resistance and TB in patients with past medical history of TB have been reported. This is the reason why Xpert MTB/RIF Ultra was developed, which has an improved sensitivity for the diagnosis of tuberculosis, especially in paucibacillary cases, and a decrease in false positives for rifampicin resistance; nowadays it is the most recommended molecular test by the World Health Ornanization (WHO), hoping to reduce the time to diagnosis and treatment. This review article analyzes the challenges of improving the diagnosis of tuberculosis, the characteristics and performance of Xpert MTB/RIF and Xpert MTB/RIF Ultra for pulmonary and extrapulmonary forms and for the detection of rifampicin resistance.

Keywords

tuberculosis; tuberculosis, pulmonary; molecular diagnostic techniques; rifampin.

\section{Introducción}

La tuberculosis (TB), causada por el Mycobacterium tuberculosis (MTB), es la principal causa de muerte mundial en adultos por un agente infeccioso y, desde hace varias décadas, es considerada un problema de salud global (1). La Organización Mundial de la Salud (OMS) estimó un número aproximado de 10 millones de enfermos de TB en 2018 (una tasa de incidencia de 132/100.000); sin embargo, solo se diagnosticaron y notificaron aproximadamente 7,3 millones de casos ese año. De estos, el 3,5 $\%$ se concentraba en la región de las Américas. Colombia notificó ese año 13.140 casos (una tasa de incidencia de 26/100.000). La brecha entre el número de casos esperados y el de los casos notificados alerta sobre la posibilidad de subdiagnóstico y subregistro de los casos detectados (2).

Dada la gran importancia que tiene la TB como un problema de salud pública, en 2014 se desarrolló e implementó la Estrategia para el Fin de la Tuberculosis, cuyos objetivos son reducir la tasa de mortalidad en un $95 \%$, reducir los nuevos casos en un $90 \%$ entre 2015 y 2035 y garantizar que ninguna familia enfrente costos catastróficos debidos a la TB. En las Américas se pretende disminuir la mortalidad anual de 25.100 (en 2015) a 2500 (en 2030) y la incidencia de $27,3 / 100.000$ (en 2015) a 5,5/100.000 (en 2030) $(3,4)$.

\section{Objetivo}

El objetivo de esta revisión fue evaluar la prueba molecular de reacción de la polimerasa en cadena (PCR, por sus siglas en inglés) Xpert MTB/RIF Ultra y conocer las diferencias respecto a su predecesora, en lo referente a la técnica y el rendimiento en la práctica clínica.

\section{Metodología}

Como estrategia de búsqueda se revisaron las bases de datos electrónicas Medline (Pubmed) y LILACS, para identificar la información. Se incluyeron revisiones sistemáticas, ensayos clínicos aleatorizados y estudios observacionales desde 1980 hasta diciembre de 2019, así como los datos epidemiológicos de la OMS, con los términos: tuberculosis AND ultra OR Xpert.

\section{Discusión}

¿Qué ha mejorado en el diagnóstico de la tuberculosis?

Además del subregistro de la enfermedad relacionado con la limitación al acceso a los servicios de salud, hay que destacar el subdiagnóstico que, en parte, se debe al rendimiento o a las dificultades técnicas de las pruebas disponibles. Por ejemplo, la baciloscopia de esputo por tinción de Ziehl-Neelsen, el primer examen usado para el diagnóstico, es un método de bajo costo y complejidad y está disponible en la mayoría de los laboratorios clínicos; pero su sensibilidad es de alrededor del $60 \%$, la cual mejora cuando se toman muestras seriadas, con un incremento del $10 \%$ con la segunda muestra 
y del $2 \%$ con la tercera. Se necesita una carga bacilar alta, al menos 10 . microrganismos $/ \mathrm{ml}$ en la muestra de esputo para que el bacilo se pueda ver en la microscopía; por esto en las presentaciones paucibacilares, por ejemplo, en coinfección con VIH y en niños o enfermedad no cavitada, la baciloscopia tiene un bajo rendimiento con una sensibilidad de entre el $50 \%$ y el $60 \%(5,6)$. Otra limitante es que la tinción de Ziehl-Neelsen no puede diferenciar entre MTB y otras especies de Mycobacterium (7).

El cultivo, considerado el patrón de referencia (gold standard) para el diagnóstico, tiene un límite de detección (LOD, por sus siglas en inglés) de 1 a 10 unidades formadoras de colonias (UFC) (8). Se hace en medio de cultivo sólido o líquido, pero se necesitan de 3 a 8 semanas y de 10 a 20 días para positivizarse, respectivamente (6). Además, es importante tener presente que su rendimiento es variable cuando se cultivan muestras extrapulmonares que requieren un proceso de descontaminación que afecta la viabilidad del bacilo. La sensibilidad depende del tipo de tejido, por ejemplo, en ganglio linfático, líquido cefalorraquídeo y hueso puede ser menor al $50 \%$ (7).

Las pruebas basadas en el método de amplificación de ácidos nucleicos emergieron como una alternativa para el diagnóstico de las enfermedades infecciosas, porque disminuyeron las limitaciones de las pruebas microbiológicas. La PCR es una de las más conocidas y utilizadas en el mundo. Es un proceso químico in vitro, mediado por una enzima termoestable, la ADN-polimerasa, la cual, en presencia de oligonucleótidos iniciadores, alineados en el lado 3' de la secuencia de neucleótidos que se quiere amplificar, duplica de forma complementaria los nucleótidos del ADN diana presente en la muestra que se analiza $(9,10)$. Durante la amplificación hay tres etapas. La primera es la desnaturalizción del ADN diana por calentamiento hasta $95^{\circ} \mathrm{C}$ durante 20 a 30 s, con lo cual se separan las hebras de la doble cadena de ADN que actuarán como plantillas o moldes. Después viene una etapa de enfriamiento alrededor de 50 a $60^{\circ} \mathrm{C}$ durante un tiempo aproximado de 2 min. En esta etapa los iniciadores se alinean al extremo 3' del ADN molde. Finalmente, la polimerasa comienza su acción catalítica agregando las bases nitrogenadas a su respectiva base complementaria haciendo una copia completa del molde. Este paso se hace a una temperatura de aproximadamente $72 \stackrel{\circ}{\circ}$ durante $3 \mathrm{~min}$. Este es un proceso cíclico que se lleva a cabo en un aparato termociclador que asegura las temperaturas y tiempos de manera precisa, el número de copias es exponencial y está determinado por el número de ciclos, lográndose un billón de copias con 30 ciclos $(9,11,12)$.

El número de ciclos necesarios para que la curva de amplificación aumente exponencialmente se conoce como umbral de ciclado (C., por sus siglas en inglés). Esta técnica está disponible comercialmente, pero también el proceso lo puede estandarizar cada laboratorio de forma propia o "casera", cuyo proceso tiene cuatro pasos (sujetos a modificaciones según el laboratorio y los materiales utilizados): lisis o extracción de $\mathrm{ADN}$, selección de la secuencia específica que se quiere amplificar, elección del método de amplificación y método de detección del producto amplificado. Las pruebas caseras se han utilizado y validado en países en vía de desarrollo, donde el acceso a las pruebas comerciales es difícil, por el costo o por la disponibilidad. La sensibilidad global, incluidas todas las muestras, está entre el $83,5 \%$ y el $87,2 \%$; sin embargo, disminuye cuando la baciloscopia es negativa y oscila entre el $62 \%$ y el $72,8 \%(11,13,14)$.

En un metanálisis que incluyó 84 estudios, Flores et al. (15) encontraron una sensibilidad para detectar MTB en muestras de esputo entre el $9 \%$ y el $100 \%$ y una especificidad de entre el $5 \%$ y el $100 \%$, con una heterogeneidad muy alta que no permite estimar la precisión clínica útil. También hallaron que el uso de la secuencia IS6110 y el método de PCR anidada mejoraban la precisión diagnóstica.

Otro estudio donde se evaluó el rendimiento de las PCR caseras mostró que son una opción costo-efectiva para tamizar casos con sospecha de $\mathrm{TB}$, especialmente en poblaciones con recursos limitados y alta prevalencia de la 
enfermedad. No obstante, hubo falsos negativos en cuatro casos de muestras paucibacilares con baciloscopia negativa. En India, un trabajo evaluó la sensibilidad y especificidad de la PCR con la amplificación de diferentes secuencias del bacilo y obtuvo sensibilidades del $83 \%$, del 86 $\%$, del $90 \%$ y del $95 \%$ amplificando secuencias para los genes mPB64, rpoB, devR y sdaA, respectivamente. La especificidad fue del $95 \%$, del $88 \%$, del $96 \%$ y del $96 \%$, respectivamente (16).

En general, las pruebas moleculares tipo PCR requieren condiciones operativas que no comprometan su rendimiento, el tipo y cantidad de la muestra procesada, el tamaño de la secuencia de $\mathrm{ADN}$ diana, la elección de los iniciadores y la experticia del personal que corre la prueba (17). Las pruebas de PCR han logrado una mayor detección, que se traduce en una mejoría de la sensibilidad, aunque esta disminuye en muestras con baciloscopia negativa o en muestras extrapulmonares.

Respecto a los casos multidrogorresistentes y resistentes a rifampicina, en 2018 se estimó que en el mundo se deberían notificar 484.000 casos, de los cuales solo se registraron 186.772 (39 $\%)$ e iniciaron tratamiento de segunda línea 156.071 pacientes (2). En las Américas, el estimado en 2018 fue de 11.000 casos de TB multidrogorresistentes y resistentes a rifampicina; pero solo se notificaron 4791 (43\%) e iniciaron tratamiento de segunda línea 4229 enfermos (88 $\%)$. Las pruebas de sensibilidad a medicamentos se hicieron solo en el $34 \%$ de los casos. Hubo también 150 de TB extensamente resistente en su mayoría procedentes de Perú $(\mathrm{n}=98)$ y de Brasil $(n=26)$ (3). La OMS estimó en 2018 para Colombia que se presentarían 580 casos de TB multidrogorresistentes y resistentes a rifampicina; pero se diagnosticaron solo 205 (35\%), tuvieron tratamiento de segunda línea 193 (94\%) y las pruebas de sensibilidad a medicamentos de segunda línea se hicieron solo para el $33 \%$. En 2018 Colombia notificó un solo caso de TB extensamente resistente (13).

A pesar de la implementación de estrategias para la eliminación de la TB, aún estamos lejos de esta meta; por ejemplo, en las Américas, solamente 15 países tienen baja incidencia ( $<10$ casos/100.000 habitantes) y ningún país entra en la categoría de preeliminación $(<1$ caso/100.000) o de eliminación $(<0,1$ casos/100.000) (3). Colombia tuvo una incidencia de casos notificados de 26/100.000 en 2018 (18).

Para erradicar este problema de salud pública, es necesario mejorar el diagnóstico y el tratamiento, la vigilancia epidemiológica y la investigación e implementación de nuevas herramientas, por lo que una de las recomendaciones para acelerar el avance hacia el fin de la TB en las Américas incluye mejorar o apresurar la implementación y expansión del diagnóstico temprano con las nuevas pruebas rápidas moleculares (3), de las cuales hacen parte el Xpert MTB/RIF y el Xpert MTB/RIF Ultra, ambas recomendadas por la OMS (2).

\section{Xpert MTB/RIF}

El Xpert MTB/RIF es una PCR automatizada, en tiempo real (amplificación y detección al mismo tiempo) que utiliza la plataforma tecnológica GeneXpert (Cepheid, Sunnyvale, CA, United States) y detecta en un solo paso el complejo M. tuberculosis y la resistencia a la rifampicina en un tiempo promedio de $2 \mathrm{~h}$ con mínima intervención de operarios. A diferencia de las pruebas convencionales de amplificación de ácidos nucleicos, el Xpert MTB/RIF cuenta con una unidad cerrada o cartucho dentro del que se integran el procesamiento de la muestra, la amplificación y la detección (19).

Inicialmente, la muestra se mezcla con un reactivo bactericida de la prueba comercial y se deja en reposo a temperatura ambiente durante $15 \mathrm{~min}$. Este es el único paso manual con la muestra; luego se transfieren $2 \mathrm{ml}$ de la mezcla dentro del cartucho para ejecutar los pasos restantes de la prueba, los cuales son totalmente automatizados. El reactivo utilizado tiene efecto bactericida que inactiva el MBT y elimina el riesgo de contagio durante el proceso $(20,21)$.

El principal mecanismo de resistencia antimicrobiano del MTB es la mutación de 
genes que codifican dianas para la unión de los fármacos anti-TB o de enzimas activadoras de medicamentos prodroga, principalmente en forma de polimorfismos en un nucleótido e inserciones y deleciones. El medicamento más frecuentemente afectado por mutaciones es la rifampicina, la cual se une a la subunidad beta de la ARN polimerasa, inhibiendo la elongación del ARN mensajero y bloqueando la síntesis de proteínas del MBT. La resistencia a rifampicina está mediada por mutaciones agrupadas en los codones 507 al 533 del gen que codifica la subunidad beta de la ARN polimerasa, el gen $r p o B$ (22). Las mutaciones más frecuentes son Ser531Leu, His526Tyr y Asp516Val (23). El conocimiento de estas mutaciones permite diseñar pruebas moleculares basadas en la amplificación de la secuencia nativa que sufre la mutación, de tal forma que si no amplifica con la sonda complementaria se identifica el defecto (24).

Para la detección de la resistencia a la rifampicina se utiliza la tecnología de sondas moleculares emisoras de señales luminosas (molecular beacon technology). Una sonda de estas está formada por un segmento de ADN de una sola hebra, dispuesto en forma de asa, que contiene la secuencia complementaria de la diana de interés (gen rpoB nativo), un elemento espaciador, un fluoróforo que emite la señal luminosa y un supresor del fluoróforo. Cuando la sonda encuentra su secuencia complementaria en la muestra, se hibrida, el asa se desenvuelve y alarga de tal forma que el fotóforo y el supresor se separa, emitiéndose una señal fluorescente. El gen rpoB nativo determina la susceptibilidad del MBT a la rifampicina y sus mutaciones implican resistencia al fármaco. La prueba utiliza 5 sondas luminosas con secuencias diferentes que conforman el gen nativo; si una de estas sondas no se une a su secuencia complementaria del gen nativo o lo hace tardíamente, con una diferencia en el C. (delta-C.) mayor de 4 ciclos, con relación a las otras sondas, significa que hay mutación en esa secuencia explorada por la sonda y una potencial resistencia a la rifampicina $(19,21)$.

La plataforma GeneXpert es un sistema lanzado en 2004 que simplificó las pruebas moleculares, al integrar la amplificación y la detección del material genético. En términos generales, consta de un módulo termociclador donde se surten todos los procesos con el cartucho que contiene la muestra, un computador con software precargado, un escáner de código de barras y un láser detector del color/ fluorescencia. El desarrollo del Xpert MTB/RIF para esta plataforma se completó en 2009 y actualmente se considera un hito en la lucha contra la TB porque, dada su portabilidad, ha permitido hacer pruebas fuera de los laboratorios clínicos especializados (25). La prueba tiene un LOD en esputo de 131 UFC (26).

El resultado de la prueba puede ser categorizado en MTB no detectado; MTB detectado con resistencia a la rifampicina; MTB detectado, sin resistencia a la rifampicina; MTB detectado con resistencia a la rifampicina indeterminada, y resultado inválido (19).

Es la prueba diagnóstica rápida más utilizada globalmente y avalada por la OMS. Entre 2010, cuando la OMS recomendó su uso, y 2017, el sector público ha aportado 9449 equipos GeneXpert y 34,4 millones de cartuchos a 130 de los 145 países elegibles para la concesión (2).

En las Américas se ha venido expandiendo la infraestructura necesaria: se pasó de 11 equipos en 2011 a más de 400 en 2017; sin embargo, solo el $13 \%$ de los casos diagnosticados ese año se hicieron por este método. En Colombia, se calcula que hay 2 a 4 equipos de GeneXpert por cada 100 casos de TB notificados (3).

Pereira et al. (27) hicieron un estudio para evaluar el impacto del Xpert MTB/RIF en el diagnóstico de la TB en una ciudad con alta prevalencia en Brasil. Encontraron que el tiempo hasta el diagnóstico fue más corto: 0,7 días con Xpert MTB/RIF versus 2 días sin uso de Xpert MTB/RIF; $p<0,0001$.

En un trabajo de Pinto et al. (28), llevado a cabo en Brasil, en el que se estimó la costoefectividad del uso de Xpert como sustituto de dos baciloscopias en el diagnóstico de TB sensible, se concluyó que en países con baja prevalencia de TB se podría esperar un beneficio económico, siempre y cuando la preocupación por los falsos negativos fuera realmente baja. Con 
lo anterior se podrían disminuir los tratamientos anti-TB iniciados empíricamente.

Calligaro et al. (29), en un estudio aleatorizado multicéntrico, evaluaron el rendimiento de Xpert MTB/RIF versus una aproximación de diagnóstico estándar (baciloscopia y cultivo de esputo) en programas de búsqueda intensiva en una población con alta prevalencia de coinfección de TB y VIH. Los autores encontraron que el uso del Xpert MTB/RIF se asoció con un inicio más temprano del tratamiento, con una reducción del tiempo de inicio del $50 \%$ a 60 días. Lawn et al. (30) hallaron que el uso del Xpert MTB/RIF, en pacientes con infección por VIH en programas de tamización intensiva, se relacionó con un aumento en la detección de casos de TB en un $45 \%$ al compararse con la baciloscopia, aunque con un desempeño subóptimo, en cuanto a la detección de resistencia a rifampicina. Cox et al. (31), en Sudáfrica, detectaron beneficios similares cuando se utilizó la prueba en pacientes con VIH en clínicas de atención primaria, lo que permite un inicio más temprano del tratamiento en comparación con el uso de la baciloscopia.

Dentro de las limitaciones de esta prueba se encuentran el bajo rendimiento en las muestras extrapulmonares paucibacilares y pulmonares con baciloscopia negativa, con una sensibilidad de alrededor el $67 \%$ (32). Los falsos positivos en pacientes con antecedente de TB tratada es otra limitante; por ejemplo, en el estudio de Theron et al. (33), 16 de 229 (7\%) muestras de pacientes diagnosticados con Xpert MTB/ RIF y tratados exitosamente tuvieron positividad en la prueba atribuida a la presencia de bacilos muertos en la muestra. Se ha estimado que el Xpert MTB/RIF tiene un $17 \%$ de falsos positivos y cerca de un $2 \%$ de falsos negativos (34). Boehme et al. (20) evaluaron el desempeño de las pruebas moleculares en el diagnóstico de $\mathrm{TB}$ y demostraron que en una cohorte la sensibilidad del Xpert MTB/RIF fue del 97 $\%$ y del $99 \%$ cuando se tenía el cultivo y la baciloscopia positivos, respectivamente; pero disminuyó cuando la baciloscopia fue negativa y el cultivo fue positivo. La sensibilidad del Xpert
MTB/RIF en 3 muestras seriadas fue del $72 \%$, del $85 \%$ y del $90 \%$, respectivamente.

Respecto a la detección de la resistencia a la rifampicina, se han descrito resultados falsos positivos en muestras mezcladas y en muestras paucibacilares, debido a retrasos en la generación de la señal en tiempo real de algunas de las sondas que utiliza la prueba. La prueba también resulta positiva cuando detecta la mutación silente, no funcional rpob F514F. Algunos estudios han mostrado una capacidad disminuida para detectar la mutación rpoB C533G, responsable de algunos casos de resistencia a rifampicina (32). Pandey et al. (35) documentaron una sensibilidad del 98,6\%, un valor predictivo negativo del 93,8 $\%$ y una especificidad y un valor predictivo positivo del $100 \%$ para el Xpert MTB/RIF cuando se evaluó la detección de resistencia a rifampicina en comparación con los métodos de susceptibilidad estándar. Para resolver estas limitaciones, se desarrolló el Xpert MTB/RIF Ultra (tabla 1).

\section{Tabla 1}

Comparación entre las características del Xpert MTBRIF y el Xpert MTBRIF Ultra

\begin{tabular}{|l|l|l|}
\hline \multicolumn{1}{|c|}{ Caracteristicas } & \multicolumn{1}{|c|}{ Xpert MTB/RIF (16) } & Xpert MTB/RIF Ultra (26,30) \\
\hline $\begin{array}{l}\text { Capacidad de la cámara para la } \\
\text { muestra }(\mu \mathrm{L})\end{array}$ & 25 & 50 \\
\hline Objetivo de la PCR & Gen $r p o B$ & IS6110, IS1081, Gen rpoB \\
\hline Características de la PCR & $\begin{array}{l}\text { Semianidada } \\
\text { RR: sondas de Beacon }\end{array}$ & $\begin{array}{l}\text { Anidada } \\
\text { RR: temperatura de fusión, } \\
\text { Sondas de Beacon }\end{array}$ \\
\hline Duración de la prueba (min) & 120 & $60-90$ \\
\hline Categorias semicuantitativas & $\begin{array}{l}\text { Alto, medio, bajo, muy } \\
\text { bajo }\end{array}$ & $\begin{array}{l}\text { Alto, medio, bajo, muy bajo, } \\
\text { trazas }\end{array}$ \\
\hline \multicolumn{3}{|c}{ Nota:referencia bibliográfica entre paréntesis. } \\
BKbaciloscopia \\
RRresistencia a la rifampicina.
\end{tabular}

\section{Xpert MTB/RIF Ultra}

Es una prueba que utiliza la misma plataforma tecnológica de GeneXpert que su predecesor Xpert MTB/RIF. Consiste en una PCR anidada, que es una modificación de la PCR convencional, que amplifica las secuencias del $\mathrm{ADN}$ en dos rondas con dos pares de iniciadores. En una primera fase, se hace una PCR con dos iniciadores externos que amplifica una región extensa del ADN que contiene la secuencia diana; luego, en una segunda fase, este producto 
de amplificación sirve de molde para una segunda PCR con dos iniciadores internos que amplifican una región interna más pequeña. Teniendo en cuenta lo anterior, la longitud del producto de amplificación de la segunda PCR será de menor tamaño que la de la primera; esta forma de amplificación mejora la sensibilidad y especificidad del examen (36).

Incorpora dos secuencias de inserción como objetivos multicopia de amplificación (IS6110 e IS1081). Además, tiene termociclados más rápidos, mejoras en la polimerasa empleada y una cámara de reacción del ADN que permite mayor volumen $(50 \mu \mathrm{L}$ vs. $25 \mu \mathrm{L})$, lo que permite que se analice una mayor cantidad de material genético (37). Con todo lo anterior, se logró disminuir el LOD a 15,6 UFC, 8 veces menor que el de Xpert MTB/RIF, con resultados negativos reportados en aproximadamente 60 min y reportes de resistencia en cerca de $90 \mathrm{~min}$ (26).

Para mejorar la precisión en la detección de la resistencia a rifampicina, el Xpert MTB/RIF Ultra no utiliza el criterio de delta CT para definir resistencia a la rifampicina, sino que utiliza el análisis basado en el delta de temperatura de fusión (mT, de melting temperature). La mT es la temperatura a la que se hibridan o se pegan los nucleótidos de la sonda al ADN complementario de la muestra. Para esto, el Xpert MTB/RIF Ultra usa 4 sondas moleculares emisoras de luz, más largas que las usadas en Xpert-MTB/RIF, de 40 o más nucleótidos (SMB, de sloppy molecular beacon) para identificar las mutaciones en la RRDR del gen rpoB, las cuales tienen diferentes $\mathrm{mT}$ con el ADN nativo; ello permite que el software las identifique. Estas sondas identifican las mutaciones fenotípicamente silentes (Q513Q y F514F), hecho que permite que su identificación no dé un resultado falso positivo, una de las limitaciones que tiene Xpert MTB/RIF $(32,37,38)$.

La detección del complejo M. tuberculosis se define como el hallazgo de uno o ambos objetivos multicopia con un CT menor a 37 ciclos y al menos 2 de las sondas del rpoB con un CT menor a 40 ciclos (32).
El Xpert MTB/RIF Ultra utiliza las mismas categorías semicuantitativas del Xpert MTB/RIF y agrega la categoría trazas, que corresponde a la menor carga genética bacilar detectable por la prueba y que se incluyó para mejorar su sensibilidad. Esta nueva categoría se reporta cuando se encuentra uno o ambos objetivos multicopia y no más de una de las sondas que tienen como objetivo el rpoB. Respecto a su interpretación, la OMS recomienda tomarlo como verdadero positivo en pacientes con infección por $\mathrm{VIH}$, en niños y en muestras extrapulmonares; sin embargo, en el resto de los casos, se debe analizar una nueva muestra para tomar decisiones clínicas. Si el segundo espécimen se informa como trazas, se deberá interpretar como TB pulmonar excepto si hay antecedente reciente de la enfermedad (29).

En cuanto a la resistencia a rifampicina, se considera ausente si se encuentra el bacilo (excepto en la categoría trazas) y todas las sondas del rpoB tienen $\mathrm{mT}$ identificables con los patrones de la secuencia nativa del gen. Para un resultado de resistencia detectable, se requiere la identificación del bacilo (IS6110, IS1081), con las 4 sondas del rpoB identificables y, al menos, una de ellas con un delta de $\mathrm{mT}$ indicativo de mutación. Si la PCR se reporta con la categoría trazas, la resistencia siempre será informada como indeterminada. A todo paciente con bajo riesgo de resistencia a la rifampicina con resultado positivo para resistencia, el Xpert MTB/RIF Ultra se debe repetir en una nueva muestra para confirmar. Cuando hay resistencia a rifampicina, es mandatorio hacer pruebas para explorar si hay resistencia a otros fármacos $(32,37)$.

Sensibilidad y especificidad de la prueba Xpert MTB/RIF Ultra para tuberculosis pulmonar

Los análisis comparativos iniciales respecto al LOD se hicieron mediante diluciones seriadas de UFC cuantificadas en muestras de esputo con la cepa $\mathrm{H} 37 \mathrm{Rv}$ del MTB que tiene 16 copias de la IS6110 y 5 copias de la IS1081. Para esta cepa, el Xpert MTB/RIF y el Xpert MTB/ RIF Ultra detectaron la bacteria y la presencia 
de la resistencia a rifampicina en el $100 \%$ de los casos con diluciones hasta de 200 UFC. Cuando se bajaban las UFC, el Xpert MTB/ RIF tuvo una sensibilidad del $85 \%$, del $50 \%$ y del $10 \%$ para 100 UFC, 50 UFC y 25 UFC, respectivamente, calculándose un LOD de 112,6 UFC; mientras que en el caso del Xpert MTB/RIF Ultra, la sensibilidad fue del $100 \%$ cuando las UFC eran mayores o iguales a 25, lo que permitió extrapolar un LOD de 15,6 UFC. En muestras con 2,5 UFC se reportó una sensibilidad del 48 $\%$ (32). Esto implica una mejoría considerable de la sensibilidad, teniendo en cuenta el LOD del cultivo (8).

El Xpert MTB/RIF Ultra también demostró una mejoría en el LOD del Mycobacterium bovis BCG que, en contraste con la cepa $\mathrm{H} 37 \mathrm{Rv}$, tiene 1 sola copia del IS6110 y 5 copias del IS1081. Para este caso, el LOD con el Xpert MTB/RIF fue de 344,1 UFC; entre tanto, el LOD con el Xpert MTB/RIF Ultra fue de 143,4 UFC (32).

Dorman et al. (39) llevaron a cabo un estudio multicéntrico en distintos escenarios geográficos para comparar el rendimiento de ambas pruebas en el diagnóstico de TB pulmonar y en la identificación de la resistencia a la rifampicina. Hallaron que el Xpert MTB/RIF Ultra no fue inferior al Xpert MTB/RIF cuando se compararon en todas las muestras con cultivo positivo, incluso fue superior en muestras con baciloscopia negativa, sensibilidad del $63 \%$ vs. un $46 \%$ (39). No obstante, encontraron una caída de la especificidad del $98 \%$ al $96 \%$, lo cual se dio en pacientes con antecedente de tuberculosis en los 7 años previos a la toma de la muestra, y después de este tiempo no se observó la diferencia. En un análisis post hoc se evaluó el efecto sobre la especificidad al reclasificar los resultados positivos de la subcategoría trazas como resultados negativos, y ello evidenció un aumento de la especificidad igual a la del Xpert MTB/RIF.

En virtud de estos resultados de sensibilidad y especificidad, los efectos del Xpert MTB/RIF Ultra respecto a los de Xpert MTB/RIF se traducen en 25 pacientes más que se diagnostican tempranamente, pero a expensas de 26 pacientes más con resultados falsos positivos (40).
En una revisión sistemática de Cochrane se documentó que el Xpert MTB/RIF Ultra tiene una sensibilidad del $88 \%$ y una especificidad del $96 \%$; mientras que Xpert MTB/RIF cuenta con valores del $83 \%$ y del $98 \%$, respectivamente. Para pacientes con infección por VIH, en ese mismo trabajo se describió que el Xpert MTB/ RIF tiene una sensibilidad del $81 \%$ y una especificidad del $98 \%$ (34). Solo el trabajo de Dorman et al. (39) ha reportado el rendimiento del Xpert MTB/RIF Ultra en esta población, con sensibilidad del $90 \%$.

Finalmente, Zhang et al. (41), mediante una revisión sistemática y metanálisis, evaluaron la precisión del Xpert MTB/RIF Ultra y encontraron una sensibilidad del 87,2\%, una especificidad del 96,5\%, una razón de verosimilitud positiva de 25,13, una razón de verosimilitud negativa de 0,13 y un área bajo la curva de 0,98 para el diagnóstico de TB pulmonar. Teniendo en cuenta estas características, calcularon una probabilidad postest para un resultado positivo del $89 \%$ y del $4 \%$ para un resultado negativo en escenarios con baja sospecha (menor al $25 \%$ ) de TB. Respecto a casos con alta sospecha (mayor al $75 \%$ ), calcularon una probabilidad postest del $99 \%$ para un resultado positivo y del $28 \%$ para un resultado negativo.

Un estudio de modelamiento con tres poblaciones hipotéticas (Sudáfrica, China e India), con diferentes prevalencias de la enfermedad, estimó el impacto en el número de tratamientos innecesarios generados, las muertes evitadas por $\mathrm{TB}$ y el número de terapias innecesarias por muerte evitada en un escenario dentro del cual se remplazara el Xpert MTB/RIF por el Xpert MTB/RIF Ultra. Para los investigadores hubo diferentes resultados dependiendo del escenario clínico: en contextos con alta prevalencia de TB e infección por VIH, es probable que el Xpert MTB/RIF Ultra ofrezca un beneficio considerable en la mortalidad; mientras que en lugares de baja prevalencia es posible que se vea un sobretratamiento. Con lo anterior, los autores concluyeron que la implementación del Xpert MTB/RIF Ultra debe darse en contextos específicos, dándole prioridad 
a las poblaciones con una prevalencia alta de TB (42).

Mishra et al. (43), recientemente, publicaron un estudio en el que evaluaron el desempeño del Xpert MTB/RIF Ultra respecto al Xpert MTB/ RIF en una población endémica de VIH en la que la infección por TB previa es frecuente; esto en dos cohortes de pacientes, en la primera adultos con sospecha clínica de TB y en la segunda pacientes con TB presuntiva y antecedente de TB en los últimos dos años. Los investigadores encontraron que en este contexto de alta carga de TB previa, el Xpert MTB/RIF Ultra generó más resultados inválidos y tuvo menor especificidad respecto al Xpert MTB/RIF. Además, en los pacientes con antecedente reciente de $\mathrm{TB}$, una cuarta parte de las muestras tuvo resultados indeterminados para la resistencia a rifampicina y el cultivo fue negativo. Ello sugiere que una prueba adicional de resistencia podría no ser exitosa.

Con los hallazgos de este estudio, Bonnet (44) destaca que la pérdida de la especificidad del Xpert MTB/RIF Ultra en los pacientes con antecedente de TB tratada es una limitación aceptable para aumentar la detección temprana de TB en pacientes con alto riesgo de desenlaces adversos, como aquellos con infección por VIH. Sin embargo, destaca es necesaria una mayor evaluación de las características y desenlaces en pacientes sintomáticos ya tratados para $\mathrm{TB}$ que tienen resultado de trazas con el objetivo de poder distinguir entre quienes requieren estudios adicionales y quienes requieren inicio de tratamiento inmediato. Hasta que se tenga mayor claridad, la decisión individualizada de tratar individuos con trazas según el antecedente de TB, las comorbilidades y los resultados de una segunda prueba (conducta recomendada cuando se obtiene el resultado trazas) es un reto para la implementación Xpert MTB/RIF Ultra en los escenarios con carga de TB, sobre todo cuando se use como primera línea de diagnóstico en centros de salud no especializados. Finalmente, resalta otra debilidad de la prueba, su incapacidad para determinar la resistencia a la rifampicina en muestras con el resultado trazas, lo que cual será un desafío para los clínicos en pacientes con antecedente de TB tratada, quienes tienen mayor riesgo de cepas resistentes.

\section{Rendimiento diagnóstico de la prueba Xpert MTB/ RIF Ultra en tuberculosis extrapulmonar}

En 2018, la tuberculosis extrapulmonar representó el $15 \%$ de los casos de TB a nivel mundial. En nuestra región aportó el 15 $\%$ (2) y, en el caso de Colombia, los casos extrapulmonares en 2018 fueron el $18 \%$ de todos los casos nuevos y recaídas (18).

El diagnóstico de la TB extrapulmonar es un gran reto diagnóstico, debido a su curso subclínico e inespecífico, a las bajas tasas de pruebas bacteriológicas positivas y a la dificultad para obtener muestras patológicas adecuadas. En el estudio prospectivo realizado por Wu et al. (45) se comparó la capacidad diagnóstica del Xpert MTB/RIF Ultra con la del Xpert MTB/ RIF y el cultivo. Encontró mejor sensibilidad del Xpert MTB/RIF Ultra en muestras con cultivo positivo $(83,7 \%$ vs. $67,4 \%$; $\mathrm{p}<0,0001)$ y con cultivo negativo (43,9\% vs. $24,84 \%)$. Cuando se evaluaron todos los especímenes, el Xpert MTB/RIF Ultra mostró mejor sensibilidad que el Xpert MTB/RIF y que el cultivo (52,5\% vs. 34 $\%$ vs. $21,5 \%$, respectivamente), con una mejor área bajo la curva para el diagnóstico de la TB extrapulmonar $(0,723$ vs. 0,650 vs. 0,608$)$. Como limitaciones hay que tener en cuenta que la mayoría de las muestras fueron líquido pleural y biopsias por aguja fina.

Pérez-Risco et al. (46) evaluaron la efectividad del Xpert MTB/RIF Ultra para la detección directa del ADN del M. tuberculosis en muestras extrapulmonares con baciloscopia negativa y hallaron una sensibilidad del $75,9 \%$ y una especificidad del $100 \%$, mejores que con el Xpert MTB/RIF. Además, se destaca la mejoría en la sensibilidad para muestras de ganglio linfático (94,1\%: un incremento entre el $17,1 \%$ y el 23,5 $\%$ respecto a informes previos con el Xpert MTB/ RIF) y para muestras de otros tejidos (86,6\%: un incremento entre el $20 \%$ y el $44,9 \%$ respecto a informes previos con el Xpert MTB/RIF) (46). El metanálisis de Zhang et al. (41) encontró 
una sensibilidad del $85,1 \%$ y una especificidad del 95,1\% para el Xpert MTB/RIF Ultra en TB extrapulmonar (41).

Con respecto a la TB pleural, que es el segundo sitio más frecuente de infección extrapulmonar después de la TB ganglionar, se ha evaluado el Xpert MTB/RIF en muestras pleurales, dada la dificultad existente para la confirmación bacteriológica, y se ha encontrado muy baja sensibilidad, $13,7 \%$ (47). Una revisión sistemática en Cochrane que evaluó el rendimiento del Xpert MTB/RIF en muestras extrapulmonares documentó una sensibilidad del $50,9 \%$ y una especificidad del $99,2 \%$ en muestras pleurales (48).

Pérez-Risco et al. (46) reportaron una sensibilidad del Xpert MTB/RIF Ultra en líquido pleural del $43,7 \%$, que fue mejor que la del cultivo $(22,3 \%)$ y que la del Xpert MTB/RIF (20,4\%). Por su parte, Wang et al. (49) también evaluaron el rendimiento del Xpert MTB/RIF Ultra para el diagnóstico de la TB pleural y tuvieron resultados similares, con sensibilidad del 44,23\% para el Xpert MTB/RIF Ultra, del 26,44 $\%$ para el cultivo, del 19,23\% para Xpert MTB/ RIF y del 1,44\% para la baciloscopia; valores altos de adenosina deaminasa y bajos valores de glucosa en el líquido pleural se asociaron con un resultado positivo del Xpert MTB/RIF Ultra. Además, reportaron una especificidad para el Xpert MTB/RIF y el Xpert MTB/RIF Ultra del $98,81 \%$ y del $100 \%$ para el cultivo y la baciloscopia positivos.

Con respecto a la biopsia pleural, Akhter et al. (50) evaluaron el rendimiento del Xpert MTB/ RIF en el tejido. Así pudieron documentar una sensibilidad del 52,2\% contra un $41 \%$ para el cultivo de la pleura. Teniendo en cuenta los resultados de los estudios en líquido pleural, que mostraron mejor rendimiento del Xpert MTB/RIF Ultra por encima del Xpert MTB/ RIF, es posible que los estudios con el Xpert MTB/RIF Ultra en tejido pleural evidencien un rendimiento mayor que el Xpert MTB/RIF y, de esta forma, faciliten el diagnóstico de la TB pleural.
Xpert MTB/RIF Ultra y resistencia a rifampicina

La rifampicina es uno de los medicamentos más efectivos contra la tuberculosis, ya que es activa contra bacilos metabólicamente activos y aquellos de metabolismo lento. La monorresistencia a este antibiótico es infrecuente y, por lo general, ocurre en conjunción a otros medicamentos, sobre todo a la isoniazida, lo que hace que la resistencia a rifampicina sea un marcador subrogado del fenotipo MDR (22).

En el estudio original de Chakravorty et al. (32), el LOD de la resistencia a rifampicina fue similar entre ambas pruebas, con 112,6 UFC para el Xpert MTB/RIF y 105,4 UFC para el Xpert MTB/RIF Ultra, pero a concentraciones por debajo de este límite, el Ultra mostró más resultados positivos (32).

Así mismo, se describió una tasa de falsos positivos del 2,7\% para el Xpert MTB/RIF y del $0 \%$ para el Xpert MTB/RIF Ultra. Este autor también evaluó la capacidad de detectar la heterorresistencia al probar mezclas de ADN nativo y $\mathrm{ADN}$ con la mutación rpoB S531L, que es la más común de las mutaciones de la RRDR. Una de las sondas SMB fue diseñada específicamente para generar valores más altos de $\mathrm{mT}$ ante la presencia de esta mutación. Con esto se encontró que el Xpert MTB/RIF Ultra tiene una capacidad para identificar la mutación cuando está presente en menos del $10 \%$ de la muestra e incluso cuando la mutación es del 5 $\%$, situación en la cual la prueba da un resultado positivo para detección de resistencia (32).

Una revisión sistemática de Cochrane indicó que el Xpert MTB/RIF tiene una sensibilidad del $85 \%$, una especificidad del $98 \%$, una tasa de falsos positivos del $16 \%$ y una tasa de falsos negativos del 0,4\% para la detección de resistencia a la rifampicina; mientras que el Xpert MTB/RIF Ultra tiene sensibilidad del $95 \%$ y una especificidad del $98 \%$, lo cual se ve reflejado en un mejor abordaje diagnóstico (34). 


\section{Conclusiones}

Las pruebas moleculares han mejorado el diagnóstico de la TB pulmonar, tal como se ha visto con el Xpert MTB/RIF desde su implementación, que permite la confirmación e inicio oportuno de la enfermedad. Aunque el diagnóstico de la TB extrapulmonar también ha mejorado con la PCR GenXpert, particularmente con Ultra, se requiere más evidencia y experiencia global para que sea una recomendación universal que permita su implementación en el escenario clínico. Los resultados de los estudios mencionados aquí, como los de Brasil y Sudáfrica, permiten extrapolar que en un escenario como el colombiano la implementación del GenXpert MTB/RIF Ultra en el diagnóstico de TB pulmonar y extrrapulmonar también daría resultados útiles; sin embargo, necesitamos hacer investigación local, lo cual es una oportunidad para los medios académicos universitarios del país.

Con la llegada del Xpert MTB/RIF Ultra y teniendo en cuenta sus ventajas respecto a su predecesor (tablas 1 y 2 ), se espera una mejoría en la toma de decisiones clínicas que ayude a cumplir con los objetivos de la Estrategia Fin de la Tuberculosis; pero para lograr esto es necesario hacer una rápida implementación de esta tecnología en todos los ámbitos de atención, sobre todo en los sitios con recursos más limitados donde el diagnóstico sigue recayendo en la baciloscopia y el cultivo.
Tabla 2

Desempeño comparativo entre el Xpert MTBRIF y el Xpert MTBRIF Ultra

\begin{tabular}{|c|c|c|c|c|}
\hline \multirow{3}{*}{$\begin{array}{l}\text { Sensibilidad TB } \\
\text { pulmonar (39) }\end{array}$} & & XPERT MTB/RIF & \multirow{2}{*}{\multicolumn{2}{|c|}{ XPERT MTB/RIF Ultra }} \\
\hline & $\mathrm{BK}$ cultivo ${ }^{+}$ & 83 & & \\
\hline & BK $(-)$ cultivo + & 46 & & 63 \\
\hline \multirow{2}{*}{\multicolumn{2}{|c|}{ Especificidad TB pulmonar }} & \multirow{2}{*}{$97,3-100 \%(8)$} & \begin{tabular}{|r|} 
Trazas + \\
$(39)$ \\
\end{tabular} & 96 \\
\hline & & & $\begin{array}{r}\text { Trazas- } \\
(39) \\
\end{array}$ & 98 \\
\hline \multirow{2}{*}{$\begin{array}{l}\text { Pacientes VIH positivo } \\
\text { (8) }\end{array}$} & \begin{tabular}{|l} 
Sensibilidad \\
\end{tabular} & $76,5-89,5$ & & $88,2-90$ \\
\hline & Especificidad & 100 & & 94,7 \\
\hline \multirow{2}{*}{ TB extrapulmonar (45) } & Sensibilidad & 67,4 & & 83,7 \\
\hline & Especificidad & 96 & & 92 \\
\hline \multirow{2}{*}{$\begin{array}{l}\text { Resistencia a } \\
\text { rifampicina (39) }\end{array}$} & Sensibilidad & 95 & & 95 \\
\hline & Especificidad & 98 & & 98 \\
\hline
\end{tabular}

NotaReferencia bibliográfica entre paréntesis. BKbaciloscopia trazas + categoría trazas tomada como TB detectada trazas - categoría trazas tomada como TB no detectada.

El entorno clínico en el cual el Xpert MTB/ RIF Ultra ha mostrado ser más sensible y eficiente para el diagnóstico de la TB ha sido en tejidos extrapulmonares, con evidencia aún en construcción sobre su eficacia en muestras con baciloscopia negativa.

Se deben hacer estudios para confirmar los beneficios del Xpert MTB/RIF Ultra sugeridos por estudios pequeños en el abordaje clínico de la TB extrapulmonar y otros casos paucibacilares, donde es imprescindible contar con mejores pruebas diagnósticas. Además, es necesario hacer estudios en escenarios reales para confirmar hallazgos como el encontrado en los estudios de modelamiento, que sugieren que la implementación del Xpert MTB/RIF Ultra en poblaciones de alta prevalencia de TB podría implicar beneficios en la mortalidad.

Con respecto a la resistencia a rifampicina, el Xpert MTB/RIF Ultra demostró una menor tasa de falsos positivos, atribuible a las mejoras en la técnica, lo cual podría evitar iniciar esquemas de segunda línea en pacientes que no lo necesitan.

\section{Conflicto de intereses}

Ninguno de los autores reporta conflicto de intereses. 


\section{Referencias}

1. Furin J, Cox H, Pai M. Tuberculosis. Lancet. 20 de abril de 2019;393(10181):1642-56.

2. World Health Organization. Global tuberculosis report 2019 [internet]. Geneva; 2019. Disponible en: https://apps.who.int/iris/bitstream/han dle/10665/329368/9789241565714-eng.pd $\mathrm{f}$

3. Organización Panamericana de la Salud. Tuberculosis en las Américas 2018 [internet]. Disponible en: https://www.paho.org/hq/index.php?op tion $=$ com_topics\&view $=$ article $\& i d=59$ $\&$ Itemid $=40776 \&$ lang $=\mathrm{es}$

4. World Health Organization. Implementing the end TB strategy: the essentials [internet]. 2015. Disponible en: https://www.who.int/tb/publications/20 15/end_tb_essential.pdf?ua $=1$

5. Dheda K, Barry CE, Maartens G. Tuberculosis. Lancet. 2016;387(10024):1211-26. https:// doi.org/10.1016/S0140-6736(15)00151-8

6. Fitzgeral D, Sterling T, Haas D. Mycobacterium tuberculosis. En: Mandell, Douglas and Bennett's principles and practice of infectious diseases. 9. a ed. New York: Elsevier; 2020. p. 2985.

7. Ríos SC, Suárez NA, Marín JG. Diagnóstico de tuberculosis pulmonar y extrapulmonar mediante un método de PCR casera en muestras clínicas de una región de desarrollo intermedio. Infectio [internet]. 2012 [citado 2020 ago 6];15(3). Disponible en: https://www.revistainfectio.org/index.p hp/infectio/article/view/454

8. Opota O, Mazza-Stalder J, Greub G, Jaton K. The rapid molecular test Xpert MTB/RIF ultra: towards improved tuberculosis diagnosis and rifampicin resistance detection. Clin Microbiol Infect. 2019;25(11):1370-6. https://doi.org/10.101 6/j.cmi.2019.03.021

9. Tamay de Dios L, Ibarra C, Velasquillo C. Fundamentos de la reacción en cadena de la polimerasa (PCR) y de la PCR en tiempo real. Investigación en
Discapacidad [internet]. 2013;2(2):70-8. Disponible en: https://www.medigraphic.co m/pdfs/invdis/ir-2013/ir132d.pdf

10. Yang S, Rothman RE. PCR-based diagnostics for infectious diseases: uses, limitations, and future applications in acute-care settings. Lancet Infect Dis. 2004;4(6):337-48. https://doi.org/10.1016/ S1473-3099(04)01044-8.

11. Sandin RL. Molecular biology of infectious diseases. En: McClatchey KD, editor. Clinical laboratory medicine. 2..$^{\mathrm{a}}$ ed. Philadelphia: Lippincott Williams \& Wilkins; 2002. p. 189-210.

12. Nolte FS. molecular techniques for diagnosis of infectious diseases. En: McClatchey KD, editor. Clinical laboratory medicine. 2. ed. Philadelphia: Lippincott Williams \& Wilkins; 2002. p. 1277-86.

13. Kirschner P, Rosenau J, Springer B, Teschner K, Feldmann K, Böttger EC. Diagnosis of mycobacterial infections by nucleic acid amplification: 18-month prospective study. J Clin Microbiol. 1996;34(2):304-12.

14. Clarridge JE, Shawar RM, Shinnick TM, Plikaytis BB. Large-scale use of polymerase chain reaction for detection of Mycobacterium tuberculosis in a routine mycobacteriology laboratory. J Clin Microbiol. 1993;31(8):2049-56.

15. Flores LL, Pai M, Colford JM, Riley LW. Inhouse nucleic acid amplification tests for the detection of Mycobacterium tuberculosis in sputum specimens: meta-analysis and meta-regression. BMC Microbiol. 2005;5:55.

16. Nimesh M, Joon D, Pathak AK, Saluja D. Comparative study of diagnostic accuracy of established PCR assays and in-house developed sdaA PCR method for detection of Mycobacterium tuberculosis in symptomatic patients with pulmonary tuberculosis. J Infect. 2013;67(5):399-407.

17. Purohit M, Mustafa T. Laboratory diagnosis of extra-pulmonary tuberculosis (EPTB) in resource-constrained setting: state of the art, challenges and the need. J Clin Diagn Res. 2015;9(4):EE01-6. 
18. World Health Organization. Tuberculosis profile Colombia 2018 [internet]. [citado 2020 ago 11]. Disponible en: https://worldhealthorg.shinyapps.io/tb_ profiles/? inputs_\&lan $=\% 22 \mathrm{EN} \% 22$ \&iso 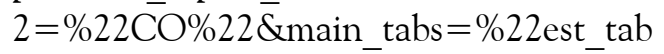 $\% 22$

19. Policy Statement: Automated Real-Time Nucleic Acid Amplification Technology for Rapid and Simultaneous Detection of Tuberculosis and Rifampicin Resistance: Xpert MTB/RIF System [internet]. Geneva: World Health Organization; 2011 [citado 2020 ago 16]. Disponible en: http:// www.ncbi.nlm.nih.gov/books/NBK304235/

20. Boehme CC, Nabeta P, Hillemann D, Nicol MP, Shenai S, Krapp F, et al. Rapid molecular detection of tuberculosis and rifampin resistance. $\mathrm{N}$ Engl J Med. 2010;363(11):1005-15.

21. Global Laboratory Initiative. Advancing TB diagnosis. GeneXpert Tecnología y Procedimientos del GeneXpert MTB/ RIF. Módulo 6. [internet]. Disponible en: https://www.paho.org/hq/dmdocument s/2016/2016-cha-genexpert-mod-6.pdf

22. Dookie N, Rambaran S, Padayatchi N, Mahomed S, Naidoo K. Evolution of drug resistance in Mycobacterium tuberculosis: a review on the molecular determinants of resistance and implications for personalized care. J Antimicrob Chemother. 01 de 2018;73(5):1138-51.

23. Desalegn Weldeyohannes DD. Review on Molecular Mechanism of First Line Antibiotic Resistance in Mycobacterium tuberculosis. Mycobact Dis [internet]. 2014 [citado 24 de agosto de 2020];04(06). Disponible en: https://www.omicsonline.org/open-acc ess/review-on-molecular-mechanism-of-fir st-line-antibiotic-resistance-in-mycobacter ium-tuberculosis-2161-1068-4-174.php?ai $\mathrm{d}=35834$

24. Goldstein BP. Resistance to rifampicin: a review. J Antibiot. 2014;67 (9):625-30.

25. Xpert MTB/RIF Implementation Manual: Technical and Operational 'How-
To'; Practical Considerations [internet]. Geneva: World Health Organization; 2014 [citado 2020 ago 16]. Disponible en: http:// www.ncbi.nlm.nih.gov/books/NBK254323/

26. Helb D, Jones M, Story E, Boehme C, Wallace E, Ho K, et al. Rapid detection of Mycobacterium tuberculosis and rifampin resistance by use of on-demand, near-patient technology. J Clin Microbiol. 2010;48(1):229-37.

27. Pereira GR, Barbosa MS, Dias NJD, de Almeida CPB, Silva DR. Impact of introduction of Xpert MTB/RIF test on tuberculosis (TB) diagnosis in a city with high TB incidence in Brazil. PLoS One. 2018;13(3). https://doi.org/10.1371/journal .pone.0193988

28. Pinto M, Steffen RE, Cobelens F, van den Hof S, Entringer A, Trajman A. Costeffectiveness of the Xpert ${ }^{\circledR}$ MTB/RIF assay for tuberculosis diagnosis in Brazil. Int J Tuberc Lung Dis. 2016;20(5):611-8.

29. Calligaro GL, Zijenah LS, Peter JG, Theron G, Buser V, McNerney R, et al. Effect of new tuberculosis diagnostic technologies on community-based intensified case finding: a multicentre randomised controlled trial. Lancet Infect Dis. 2017;17(4):441-50.

30. Lawn SD, Brooks SV, Kranzer K, Nicol MP, Whitelaw A, Vogt M, et al. Screening for HIV-associated tuberculosis and rifampicin resistance before antiretroviral therapy using the Xpert MTB/RIF assay: a prospective study. PLoS Med. 2011;8(7). https://doi.org/10.13 71/journal.pmed.1001067

31. Cox HS, Mbhele S, Mohess N, Whitelaw A, Muller O, Zemanay W, et al. Impact of Xpert MTB/RIF for TB diagnosis in a primary care clinic with high $\mathrm{TB}$ and HIV prevalence in South Africa: a pragmatic randomised trial. PLoS Med. 2014;11(11). https://doi.org/10.1371/journ al.pmed.1001760

32. Chakravorty S, Simmons AM, Rowneki M, Parmar H, Cao Y, Ryan J, et al. The New Xpert MTB/RIF ultra: improving detection of Mycobacterium tuberculosis and resistance 
to rifampin in an assay suitable for point-ofcare testing. mBio. 2017;8(4). https://doi.o $\mathrm{rg} / 10.1128 / \mathrm{mBio} .00812-17$

33. Theron G, Venter R, Smith L, Esmail A, Randall P, Sood V, et al. False-Positive Xpert MTB/RIF results in retested patients with previous tuberculosis: frequency, profile, and prospective clinical outcomes. J Clin Microbiol. 2018;56(3). https://doi.org/10.1 128/JCM.01696-17

34. Horne DJ, Kohli M, Zifodya JS, Schiller I, Dendukuri N, Tollefson D, et al. Xpert MTB/RIF and Xpert MTB/RIF Ultra for pulmonary tuberculosis and rifampicin resistance in adults. Cochrane Database Syst Rev. 2019;2019(6). https://doi.org/10. 1002/14651858.CD009593.pub4

35. Pandey P, Pant ND, Rijal KR, Shrestha B, Kattel S, Banjara MR, et al. Diagnostic accuracy of GeneXpert MTB/RIF assay in comparison to conventional drug susceptibility testing method for the diagnosis of multidrugresistant tuberculosis. PLoS ONE. 2017;12(1):e0169798. https://doi.org/10.1 371/journal.pone.0169798

36. Sandoval-Rodríguez A, Meza-Ríos A, Floresvillar-Mosqueda JF. Reacción en cadena de la polimerasa. En: Biología molecular: fundamentos y aplicaciones en las ciencias de la salud. 2. ${ }^{\text {a }}$ ed. Ciudad de México: McGraw-Hill Medical; 2016.

37. World Health Organization. WHO meeting report of a technical expert consultation: non-inferiority analysis of Xpert MTB/RIF ultra compared to Xpert MTB/RIF [internet]. 2017. Disponible en: https://apps.who.int/iris/bitstream/han dle/10665/254792/WHO-HTM-TB-2017. 04-eng.pdf? sequence $=1$ \&isAllowed $=y$

38. Chakravorty S, Kothari H, Aladegbami B, Cho EJ, Lee JS, Roh SS, et al. Rapid, highthroughput detection of rifampin resistance and heteroresistance in Mycobacterium tuberculosis by use of sloppy molecular beacon melting temperature coding. J Clin Microbiol. 2012;50(7):2194-202.
39. Dorman SE, Schumacher SG, Alland D, Nabeta P, Armstrong DT, King B, et al. Xpert MTB/RIF ultra for detection of Mycobacterium tuberculosis and rifampicin resistance: a prospective multicentre diagnostic accuracy study. Lancet Infect Dis. 2018;18(1):76-84.

40. Arend SM, van Soolingen D. Performance of Xpert MTB/RIF Ultra: a matter of dead or alive. Lancet Infect Dis. 2018;18(1):8-10.

41. Zhang M, Xue M, He J-Q. Diagnostic accuracy of the new Xpert MTB/RIF Ultra for tuberculosis disease: A preliminary systematic review and meta-analysis. Int J Infect Dis. 2020;90:35-45. https://doi.org/1 0.1016/j.ijid.2019.09.016

42. Kendall EA, Schumacher SG, Denkinger CM, Dowdy DW. Estimated clinical impact of the Xpert MTB/RIF Ultra cartridge for diagnosis of pulmonary tuberculosis: a modeling study. PLoS Med. 2017;14(12):e1002472.

43. Mishra H, Reeve BWP, Palmer Z, Caldwell J, Dolby T, Naidoo CC, et al. Xpert MTB/RIF Ultra and Xpert MTB/RIF for diagnosis of tuberculosis in an HIVendemic setting with a high burden of previous tuberculosis: a two-cohort diagnostic accuracy study. Lancet Respir Med. 2020;8(4):368-82. https://doi.org/10. 1016/S2213-2600(19)30370-4

44. Bonnet M. Xpert MTB/RIF Ultra: what is the real impact? Lancet Respir Med. 2020;8(4):325-6. https://doi.org/10.1016/S 2213-2600(20)30050-3

45. Wu X, Tan G, Gao R, Yao L, Bi D, Guo Y, et al. Assessment of the Xpert MTB/RIF Ultra assay on rapid diagnosis of extrapulmonary tuberculosis. Int J Infect Dis. 2019;81:91-6. https://doi.org/10.1016/j.ijid.2019.01.050

46. Pérez-Risco D, Rodríguez-Temporal D, Valledor-Sánchez I, Alcaide F. Evaluation of the Xpert MTB/RIF Ultra assay for direct detection of Mycobacterium tuberculosis complex in smear-negative extrapulmonary samples. J Clin Microbiol. 2018;56(9).

47. Macías A, Sánchez-Montalvá A, Salvador F, Villar A, Tórtola T, Saborit N, et 
al. Epidemiology and diagnosis of pleural tuberculosis in a low incidence country with high rate of immigrant population: a retrospective study. Int J Infect Dis. 2019;78:34-8. https://doi.org/10.1016/j.ijid .2018.10.005

48. Kohli M, Schiller I, Dendukuri N, Dheda K, Denkinger CM, Schumacher $\mathrm{SG}$, et al. Xpert ${ }^{\circledR} \mathrm{MTB} / \mathrm{RIF}$ assay for extrapulmonary tuberculosis and rifampicin resistance. Cochrane Database Syst Rev. 2018;2018(8). https://doi.org/10.1002/146 51858.CD012768.pub2

49. Wang $G$, Wang $S$, Yang $X$, Sun $Q$, Jiang G, Huang M, et al. Accuracy of Xpert MTB/RIF Ultra for the diagnosis of pleural TB in a multicenter cohort study. Chest. 2020;157 (2):268-75. https://doi.org/10.101 6/j.chest.2019.07.027

50. Akhter N, Sumalani KK, Chawla D, Ahmed Rizvi N. Comparison between the diagnostic accuracy of Xpert MTB/Rif assay and culture for pleural tuberculosis using tissue biopsy. ERJ Open Res. 2019;5(3):00065-2019. https://doi.org/10.1 183/23120541.00065-2019 